$\mathrm{n}=20$; no SIRS, $21.6(18.6-43.1) \mathrm{nmol} / \mathrm{l}, \mathrm{n}=13, \mathrm{p}<0.0001)$, and in PODs who died/required emergency liver transplantation (OLT 72.9 (68.1-119.2) nmol/1, $\mathrm{n}=14$; survived 30.3 (20.7-89.1) $\mathrm{nmol} / \mathrm{l}, \mathrm{n}=29$, $\mathrm{p}=0.006$; AUROC $78.6 \%$ (95\% CI $62.2 \%$ to $94.9 \%$ ). An admission neopterin value of $50 \mathrm{nmol} / \mathrm{l}$ predicted death/OLT with a sensitivity of 100.0 (95\% CI 76.8 to 100.0) and specificity of 63.2 (95\% CI 38.4 to 83.7). Admission neopterin levels in PODs correlated with: serum creatinine (Spearman's $\mathrm{r}=0.847, \mathrm{p}<0.0001$ ); prothrombin time $(\mathrm{r}=0.439, \mathrm{p}=0.011)$; platelet count $(\mathrm{r}=-0.463, \mathrm{p}=0.007)$; IL-6 $(\mathrm{r}=0.650, \mathrm{p}=0.0001) ; \mathrm{IL}-10 \quad(\mathrm{r}=0.529, \mathrm{p}=0.004)$; serum ferritin $(\mathrm{r}=0.467, \mathrm{p}=0.006)$; and with organ failure scores (SOFA, $r=0.725$, $\mathrm{p}<0.0001$; APACHE II, $\mathrm{r}=0.659, \mathrm{p}<0.0001)$.

Conclusion Serum neopterin levels are significantly elevated following POD and are correlated with adverse outcomes. Serum neopterin may have value as an early marker of prognosis in ALF and should be assessed further with larger prospective cohorts of ALF patients. The correlation of serum neopterin with adverse outcomes provides further support for the importance of macrophage activation in the pathogenesis of multiorgan failure in ALF.

Competing interests None declared.

\section{PTU-006 THE SEQUENTIAL ORGAN FAILURE ASSESSMENT (SOFA) SCORE IS AN EFFECTIVE TRIAGE MARKER FOLLOWING STAGGERED PARACETAMOL (ACETAMINOPHEN) OVERDOSE}

doi:10.1136/gutjnl-2012-302514c.6

${ }^{1} \mathrm{D}$ G Craig, ${ }^{*} \mathrm{~S}$ Zafar, ${ }^{2} \mathrm{~T}$ Reid, ${ }^{1} \mathrm{~J}$ Davidson, ${ }^{1} \mathrm{~K}$ Martin, ${ }^{3} \mathrm{P} \mathrm{C}$ Hayes, ${ }^{3} \mathrm{~K} \mathrm{~J}$ Simpson. ${ }^{1}$ Scottish Liver Transplantation Unit, Royal Infirmary of Edinburgh, Edinburgh, UK; ${ }^{2}$ University of Edinburgh, Edinburgh, UK; ${ }^{3}$ Division of Clinical and Surgical Sciences, University of Edinburgh, Edinburgh, UK

Introduction The Sequential Organ Failure Assessment (SOFA) score is an effective triage marker following single time point paracetamol (acetaminophen) overdose, ${ }^{1}$ but has not been evaluated following staggered paracetamol overdose. The aims of this study were to evaluate the prognostic accuracy of the SOFA score in a cohort of severe acute liver injury patients following staggered paracetamol overdose.

Methods Time-course analysis of 50 staggered paracetamol overdoses admitted to a tertiary liver centre. Individual laboratory samples were correlated with the corresponding clinical parameters in relation to time from admission, and the daily SOFA score calculated.

Results A total of $39 / 50$ (78\%) patients developed hepatic encephalopathy, and therefore acute liver failure. The area under the SOFA receiver operator characteristic for death/liver transplantation was 87.4 (95\% CI 73.2 to 95.7), 94.3 (95\% CI 82.5 to 99.1), and 98.4 $(95 \%$ CI 84.3 to 100.0$)$ at 0,24 , and $48 \mathrm{~h}$ respectively post-admission. A SOFA score of $<6$ at tertiary care admission predicted survival with a sensitivity of $100.0 \%(95 \%$ CI $76.8 \%$ to $100.0 \%)$ and specificity of $58.3 \%$ (95\% CI $40.8 \%$ to $74.5 \%$ ), compared with $85.7 \%$ ( $95 \%$ CI $60.6 \%$ to $97.4 \%$ ) and $75.0 \%$ (95\% CI $65.2 \%$ to $79.5 \%)$ respectively for the modified Kings College criteria. Only $2 / 21$ patients with an admission SOFA score $<6$ required renal replacement therapy or intracerebral pressure monitoring. SOFA significantly outperformed the Model for End-stage Liver Disease at 0 $(p=0.0013), 24 \quad(p=0.0001)$ and $48 \mathrm{~h} \quad(p=0.0193) \quad$ following admission.

Conclusion A SOFA score $<6$ at tertiary care admission following a staggered paracetamol overdose carries a high negative predictive value. The SOFA score could improve triage of high risk staggered paracetamol overdose patients.

\section{Competing interests None declared.}

\section{REFERENCE}

1. Craig DG, Reid TW, Martin KG, et al. The systemic inflammatory response syndrome and sequential organ failure assessment scores are effective triage markers following paracetamol (acetaminophen) overdose. Aliment Pharmacol Ther 2011;34:219-28.

\section{PTU-007 CEREBRAL OEDEMA IS RARE IN ACUTE-ON-CHRONIC LIVER FAILURE}

doi:10.1136/gutjnl-2012-302514c.7

${ }^{1} \mathrm{D}$ Joshi, ${ }^{1} \mathrm{~A}$ Patel, ${ }^{1} \mathrm{~J}$ O'Grady, ${ }^{1} \mathrm{D}$ Shawcross, ${ }^{2} \mathrm{~S}$ Connors, ${ }^{1} \mathrm{C}$ Willars, ${ }^{1} \mathrm{~W}$ Bernal, ${ }^{1} \mathrm{~J}$ Wendon, ${ }^{1} \mathrm{G}$ Auzinger. ${ }^{1}$ Institute of Liver Studies, King's College Hospital, London, UK; '2Department of Neuro-radiology, King's College Hospital, London, UK

Introduction Acute-on-chronic liver failure (AoCLF) has a rapidly progressive disease course associated with significant mortality. Hepatic encephalopathy (HE) is common associated with hyperammonemia, systemic inflammation and hyponatremia. The prevalence of cerebral oedema in AoCLF is unknown. We aimed to describe the prevalence of cerebral oedema in a cohort of AoCLF adult ( $>18$ years) patients admitted to the liver intensive treatment unit (LITU) between January 2005 and 2011.

Methods AoCLF was defined using criteria of Sarin et al. ${ }^{1}$ Arterial ammonia (NH3), MELD, UKELD, and organ failure (SOFA) scores were collated (results expressed as medians with ranges). Patients who had undergone cranial CT imaging were identified. Neuroimages were reported by consultant neuro-radiologists.

Results During the study period, 1008 patients with chronic liver disease (CLD) were admitted to the LITU. 173 patients (110 male) underwent neuro-imaging. Of these 81 (48 male) fulfilled criteria for AoCLF, Over the same time period 655 patients were admitted with acute liver failure. Variceal bleeding (30\%) and sepsis (31\%) were the most frequent precipitants of AoCLF. Compared to the CLD group, AoCLF patients were younger $(50,24-71$ vs $59,30-74, p=0.001)$, serum NH3 $(143,40-305$ vs $111,28-315)$, grade of $H E(3,1-4$ vs 1 , $0-4)$, MELD (25, 8-40 vs $15,6-34)$, SOFA (11, 2-17 vs 4, 0-14), UKELD (63, $50-75$ vs $55,44-73)$ and SIRS score $(2,1-3$ vs $1,0-3)$ were higher $(p<0.0001$ for all). Serum sodium was lower in the AoCLF group (132 118-154 vs 136120-146, $p<0.0001)$. HE ( $\geq$ grade 3 ) occurred in $66 \%$ of AoCLF patients vs $13 \%$ CLD ( $p<0.0001)$. In those with neuro-imaging, $26 \%$ were normal, $26 \%$ demonstrated increased cerebral atrophy for age, $15 \%$ small vessel disease and $10 \%$ intra-cranial haemorrhage. Cerebral oedema was seen in two patients with AoCLF, 1 post TIPSS (NH3 $289 \mu \mathrm{mol} / \mathrm{l}$ ) and 1 with septic shock (NH3 $268 \mu \mathrm{mol} / \mathrm{l}$ ). 72 patients with ALF underwent neuro-imaging with $32 \%$ showing radiological evidence of cerebral oedema. Compared to the CLD group, 30 and 90 day survival was poorer in AoCLF $(52 \%$ and $42 \%$ vs $80 \%$ and $75 \%$, log rank $\mathrm{p}<0.0001)$. The mode of death was that of progressive multi-organ failure (MOF). The two patients with cerebral oedema on CT suffered cerebral deaths with tonsillar herniation. AUROC analysis for survival of AoCLF identified SOFA $(0.67,95 \%$ CI 0.54 to 0.8 , $\mathrm{p}=0.02)$ MELD $(0.74,95 \%$ CI 0.61 to $0.87, \mathrm{p}<0.0001)$ and UKELD (0.87, 95\% CI 0.8 to $0.97, \mathrm{p}<0.0001)$.

Conclusion Our data demonstrates poor outcome in patients with AoCLF compared to those with CLD requiring admission to LITU. Mortality was attributable to MOF and although deep levels of encephalopathy requiring ventilation were common (66\%), the prevalence of cerebral oedema was rare at $2 \%$.

Competing interests None declared.

\section{REFERENCE}

1. Sarin, et al. Hep Int 2009. 